\title{
REACTOR GAMMA HEAT MEASUREMENTS WITH THERMOLUMINESCENT PHOSPHORS
}

J. C. TAPPENDORF

\section{MASTER}

\section{Aerojet nuclear Company}

NATIONAL REACTOR TESTING STATION Idaho Falls, Idaho - 83401

\section{U. S. ATOMIC ENERGY COMMISSION}




\section{DISCLAIMER}

This report was prepared as an account of work sponsored by an agency of the United States Government. Neither the United States Government nor any agency Thereof, nor any of their employees, makes any warranty, express or implied, or assumes any legal liability or responsibility for the accuracy, completeness, or usefulness of any information, apparatus, product, or process disclosed, or represents that its use would not infringe privately owned rights. Reference herein to any specific commercial product, process, or service by trade name, trademark, manufacturer, or otherwise does not necessarily constitute or imply its endorsement, recommendation, or favoring by the United States Government or any agency thereof. The views and opinions of authors expressed herein do not necessarily state or reflect those of the United States Government or any agency thereof. 


\section{DISCLAIMER}

Portions of this document may be illegible in electronic image products. Images are produced from the best available original document. 
Printed in the United States of America Available frum

National Technical Information Service

U. S. Department of Commerce

5285 Port Royal Road

Springfield, Virginia 22151

Price: Printed Copy \$3.00; Microfiche \$0.95

\section{LEGAL NOTICE}

This report was prepared as an account of work sponsored by the United States Government. Neither the United States nor the United States Atomic Energy Commission, nor any of their employees, nor any of their contractors, subcontractors, or their employees, makes any warranty, express or implied, or assumes any legal liability or responsibility for the accuracy, completeness or usefulness of any information, apparatus, product or process disclosed, or represents that its use would not infringe privately owned rights. 


\section{J.C. Tappendorf}

\section{AEROJET NUCLEAR COMPANY}

Date Published -- March 1972

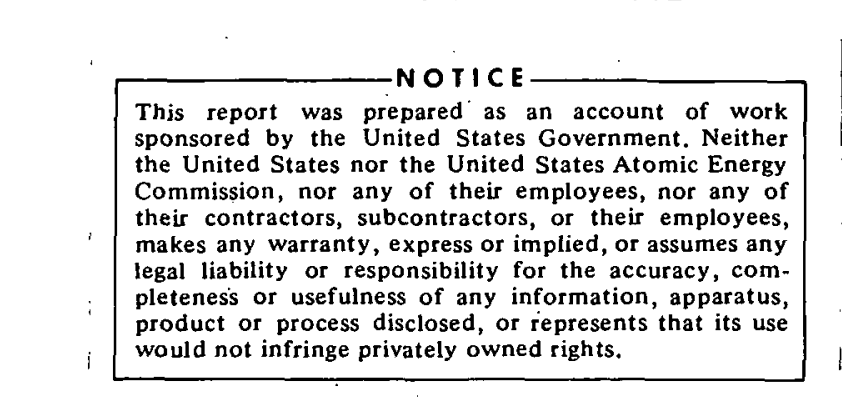

PREPARED FOR THE U. S. ATOMIC ENERGY COMMISSION IDAHO OPERATIONS OFFICF. UNDER CONTRACT No. AT(10-1)-1375 


\section{ACKNOWLEDGENTS}

The author wishes to thank J. P. Cusimano and his staff of the Dosimetry Branch, AEC Idaho Operations Office, Health Services Laboratory, for their valuable assistance and advice in applying the technology of solid state dosimetry to the measurement of gamma heat in a critical facility. Special thanks are given to B. W. Howes and C. H. Hogg for their technical advice. 


\begin{abstract}
The measurement of gamma heat in a critical facility using the thermoluminescent phosphor $\mathrm{CaF}_{2}: \mathrm{Mn}$ has proven to be a reliable means of obtaining gamma heat information for test reactors. The small size of the $\mathrm{CaF}_{2}: \mathrm{Mn}$ detectors enables detailed measurements without having to remove any reactor hardware to accommodate the detectors. Additionally, the phosphor is sufficiently sensitive for irradiations at a reactor power level of approximately 300 watts for 20 minutes. The results of the measurements are reported in terms of gamma heating in aluminum because of the similarity of the energy response to gamma rays for aluminum and the phosphor. The absolute and relative errors for this technique of measuring gamma heat for test reactors are estimated to be $\pm 21 \%$ and $\pm 5 \%$, respectively, at $95 \%$ confidence limits.
\end{abstract}




\section{CONTENTS}

ACKNOWLEDGMENTS $\ldots \ldots \ldots \ldots \ldots \ldots \ldots \ldots \ldots \ldots \ldots \ldots \ldots \ldots$

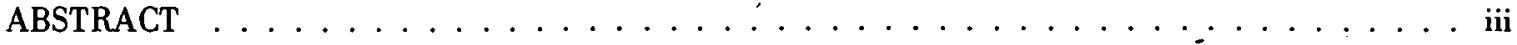

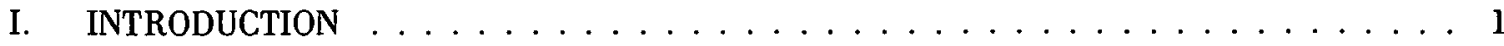

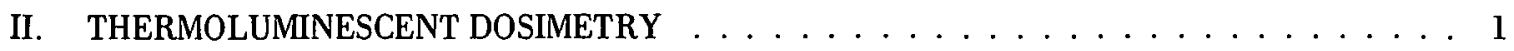

1. THERMOLUMINESCENT MECHANISMS $\ldots \ldots \ldots \ldots \ldots \ldots \ldots$

2. THERMOLUMINESCENT PHOSPHOR CHARACTERISTICS $\ldots \ldots \ldots \ldots \ldots \ldots$

2.1 Glow Curve . . . . . . . . . . . . . . . . . . 2

2.2 Thermoluminescent Emission Spectrum . . . . . . . . . . . . . 2

2.3 Retention of Stored Signal . . . . . . . . . . . . . . . . . 3

2.4 Response as a Function of Incident Gamma Energy . . . . . . . . . . . . . . . 4

2.5 Response to Thermal Neutrons . . . . . . . . . . . . . . . 6

2.6 Response of Phosphor as a Function of Exposure . . . . . . . . . . . . 6

3. THERMOLUMINESCENCE MEASURING APPARATUS $\ldots \ldots \ldots \ldots \ldots \ldots$

III. APPLICATION OF THERMOLUMINESCENT DOSIMETRY TO MEASURING GAMMA HEAT . 8

1. QUANTITATIVE DESCRIPTION OF GAMMA RAY ENERGY DEPOSITION $\ldots \ldots$. 8

2. CHARGED PARTICLE EQUILIBRIUM $\ldots \ldots \ldots \ldots \ldots$

3. CALIBRATION OF DOSIMETER RESPPONSE TO GAMMA HEAT $\ldots \ldots \ldots \ldots \ldots$

4. INTERPRETATION OF DOSIMETER READOUTS AFTER IRRADIATION IN A CRITICAL FACILITY $\ldots \ldots \ldots \ldots \ldots \ldots \ldots$

IV. TECHNIQUE FOR MEASURING GAMMA HEAT IN A CRITICAL FACILITY $\ldots \ldots$

1. $\mathrm{CaF}_{2}:$ Mn THERMOLUMINESCENT PHOSPHOR CHARACTERISTICS $\ldots \ldots \ldots \ldots$

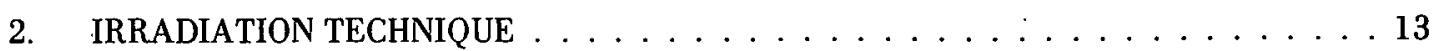

3. CORRECTIONS TO $\mathrm{CaF}_{2}$ :Mn DOSIMETER READOUTS. . . . . . . . 13

$3.1 \quad$ Fading of Stored Signal $\ldots \ldots \ldots \ldots \ldots$

3.2 Correction for Time-Varying Fission Product Source Strength $\ldots \ldots \ldots$. . . . 14

3.3 Correction for Distance from Core Source . . . . . . . . . . . . . . 14

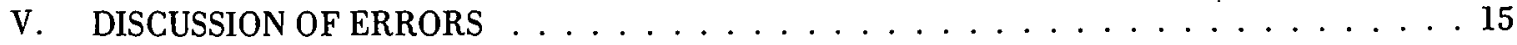

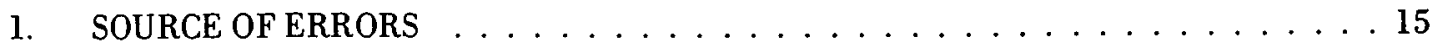

1.1 Neutron Effects . . . . . . . . . . . . . . . . . 15

1.2 Calibration Errors . . . . . . . . . . . . . . . . 16

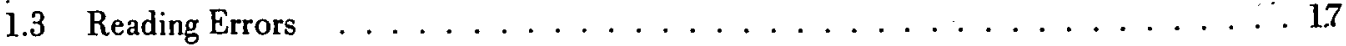


2. DETERMINATION OF RELATIVE AND ABSOLUTE ERRORS . . . . . . . 18

2.1 Relative Error . . . . . . . . . . . . . . . . . . . 18

2.2 Absolute Error . . . . . . . . . . . . . . . . . . . . . . 19

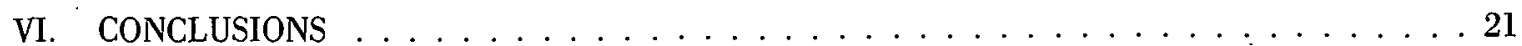

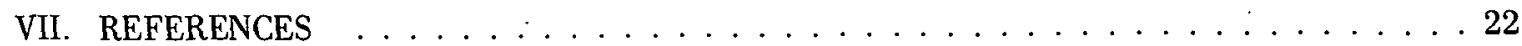

APPENDIX A -- PROCEDURE FOR USING $\mathrm{CaF}_{2}$ :Mn DOSIMETERS TO MEASURE GAMMA HEAT IN

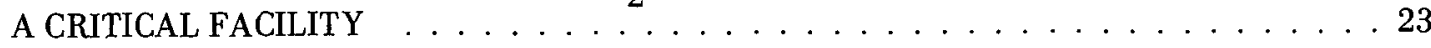

\section{FIGURES}

1. Illustration of the effect of the interaction of a

gamma or X-ray on a thermoluminescent phosphor $\ldots \ldots \ldots \ldots \ldots$

2. Relative light intensity of the thermoluminescent phosphors $\mathrm{CaF}_{2}: \mathrm{Mn}$ and $\mathrm{LiF}$ (TLD-100) as a function of phosphor temperature $\ldots \ldots \ldots \ldots \ldots \ldots \ldots \ldots$

3. Relative light intensity of the thermoluminescent phosphors $\mathrm{CaF}_{2}: \mathrm{Mn}$ and $\mathrm{LiF}$ (TLD-100) as a function of emitted radiation

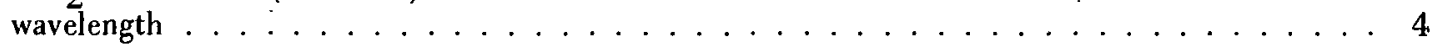

4. Relative response per roentgen of the thermoluminescent phosphors $\mathrm{CaF}_{2}: \mathrm{Mn}$ and LiF (TLD-100) as a function of

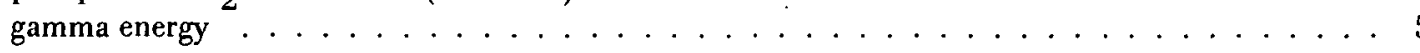

5. The relative glow peak intensity for $\mathrm{CaF}_{2}: \mathrm{Mn}$ and $\mathrm{LiF}$

(TLD-100) as a function of gamma-ray exposure

6. Schematic diagram of the principal components of a

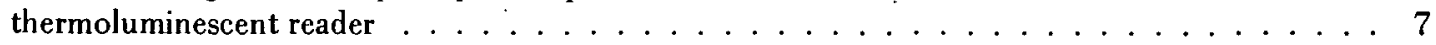

7. Illustration of the timing of a flux run to assure that the exposure to the dosimeters in the reactor is equivalent to the exposure if the flux were made at the maximum power level for the time of the irradiation

8. Illustration of physical setup for calibrating the thermoluminescence of $\mathrm{CaF}_{2}: \mathrm{Mn}$ to absorbed dose 16 


\section{TABLES}

I. Nominal Characteristics of Solid $\mathrm{CaF}_{2}: \mathrm{Mn}$ Dosimeters $\ldots \ldots \ldots \ldots \ldots \ldots \ldots \ldots \ldots$

A-I. Typical TL Analyzer Conditions for Reading of Solid $\mathrm{CaF}_{2}: \mathrm{Mn}(1 \times 1 \times 6 \mathrm{~mm})$ Dosimeters . . . 24 


\section{REACTOR GAMMA HEAT MEASUREMENTS WITH THERMOLUMINESCENT PHOSPHORS}

\section{INTRODUCTION}

Knowledge of gamma heating in the structural materials of the reactor, experiment loops, and experiments is needed to determine cooling requirements and predict the magnitude of thermal stresses. In response to past requests, gamma heat measurements were performed in the test reactors using ion chambers. Recently, many of these measurements have been performed in the test reactor critical facilities using the thermoluminescent phosphor $\mathrm{CaF}_{2}: \mathrm{Mn}$ as the gamma heat monitor. This technique significantly reduces the cost of such measurements and allows for more detailed measurements as a result of the detector size.

This report discusses the application of thermoluminescent (TL) dosimetry to measuring gamma heat in critical facilities and the extrapolation of these results to test reactor full-power conditions. Additionally, a determination of the relative and absolute errors for this measuring technique is made.

\section{THERMOLUMINESCENT DOSIMETRY}

Thermoluminescent dosimetry has recently been established as one of the important methods of measuring radiation qualities in the field of radiation dosimetry. The rapid development and the daily changes in the state-of-the-art of thermoluminescent dosimetry reveal daily additional applications for its use in radiation measurements. The application of this principle to critical facility gamma heat measurements has provided an inexpensive and versatile means of obtaining gamma heat information for test reactors.

\section{THERMOLUMINESCENT MECHANISMS}

When thermoluminescent phosphor is exposed to ionizing radiation, electrons are freed. Many of these electrons are then trapped at lattice imperfections in the phosphor. This effect is shown in Figure 1(a) for the case of gamma or $\mathrm{X}$-rays. The gamma or $\mathrm{X}$-ray either frees or creates an electron through the photoelectric, Compton, or pair-production effects and this electron is allowed to travel through the crystal in the conduction band until it is trapped. When heat is subsequently applied to the phosphor, energy is imparted to the electrons allowing the electrons to escape from the traps and, upon return to the valence band, emit light. This effect is shown in Figure 1(b). Often the phosphor is doped with known luminescent ions (such as $\mathrm{Mn}^{2+}$ ) to ensure that this process is radiative and to control the emission spectrum ${ }^{[1]}$.

The light emanating from the phosphor as it is being heated can be measured using a photomultiplier tube and associated electronics. This output is plotted as a function of the temperature of the phosphor to give a glow curve. The area underneath the glow curve is a measure of the absorbed dose in the phosphor. 


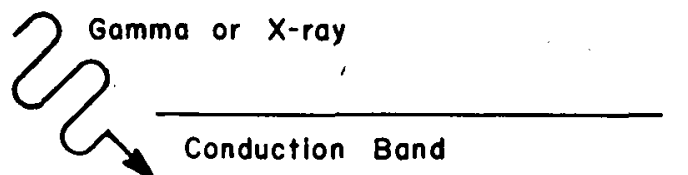

Conduction Band
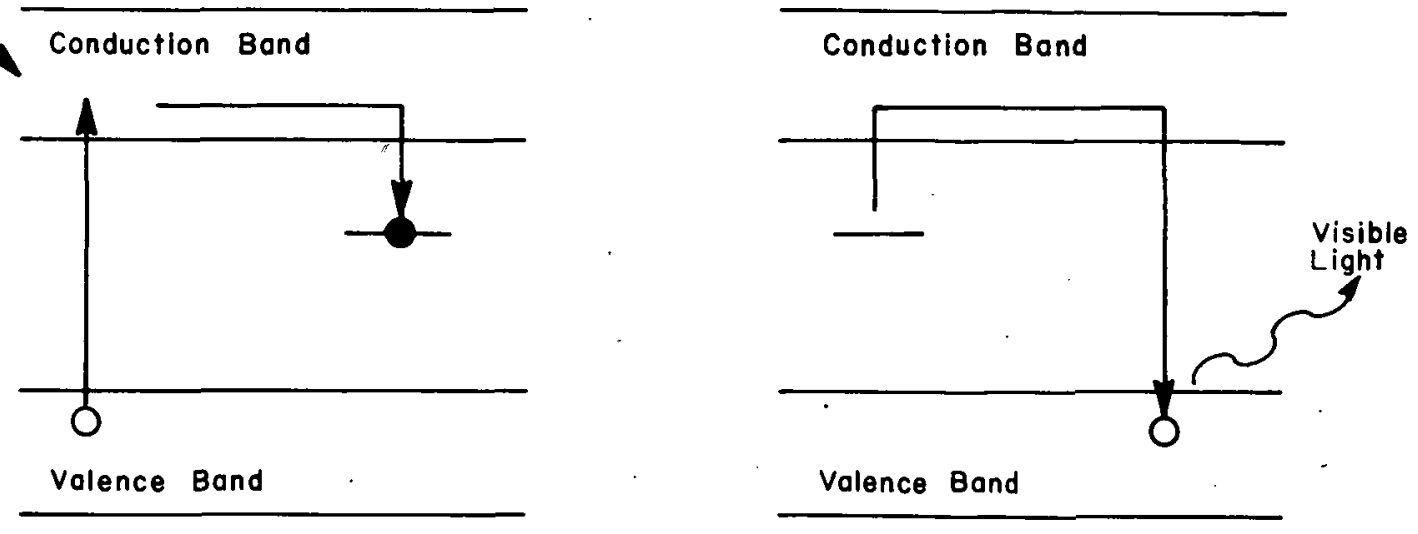

Electron

1 (o) $\quad$ Hole

1 (b)

Fig. 1 Illustration of the effect of the interaction of a gamma or X-ray on a thermoluminescent phosphor. Figure 1(a) shows the gamma or X-ray freeing an electron and the subsequent trapping of the electron at a lattice imperfection. Figure l(b) shows the freeing of the electron as a result of heating and the subsequent return to the valence band with the emission of light.

\section{THERMOLUMINESCENT PHOSPHOR CHARACTERISTICS}

There are many characteristics of a thermoluminescent phosphor to consider in deciding what phosphor to use for a particular application. In order to understand the significance of these characteristics, the characteristics of two widely used phosphors will be discussed and compared.

Two phosphors which have been widely used and their characteristics studied extensiyely are $\mathrm{LiF}$ $(\mathrm{TLD}-100)$ and $\mathrm{CaF}_{2}: \mathrm{Mn}$. The isotopic ratio for lithium in TLD-100 is approximately $92.6 \%{ }^{7} \mathrm{Li}$ and $7.4 \%$ $6 \mathrm{Li}$. Many of the characteristics of these two phosphors are quite similar. However, some of their characteristics are different enough such that they may not be used for the same application.

\subsection{Glow Curve}

After a thermoluminescent phosphor has been exposed to a gamma or X-ray source, it is heated to release the trapped electrons. This process gives off light, the intensity of which is dependent upon the temperature of the phosphor. The plot of the light intensity as a function of phosphor temperature is called a glow curve. Glow curves indicate the abundance of electrons trapped at various energy depths since the thermal energy imparted to release a trapped electron indicates the energy depth in its trapped state. Representative glow curves for $\mathrm{CaF}_{2}: \mathrm{Mn}$ and $\mathrm{LiF}$ (TLD-100) are shown in Figure $2^{[2]}$. These curves show that both phosphors have deep electron energy traps because of the high temperature at which the curves peak. Thus they both can be used and stored where temperatures are not excessive.

\subsection{Thermoluminescent Emission Spectrum}

The spectrum of the light emitted from the phosphor is another important characteristic.. Figure 3 shows the relative light intensity as a function of the wavelength of the light for $\mathrm{CaF}_{2}: \mathrm{Mn}$ and $\mathrm{LiF}$ (TLD-100) ${ }^{[2]}$.

It is desirable to use a phosphor which has a narrow radiation spectrum. The peak wavelength of this spectrum should be approximately 4000 to $5000 \mathrm{~A}$. 


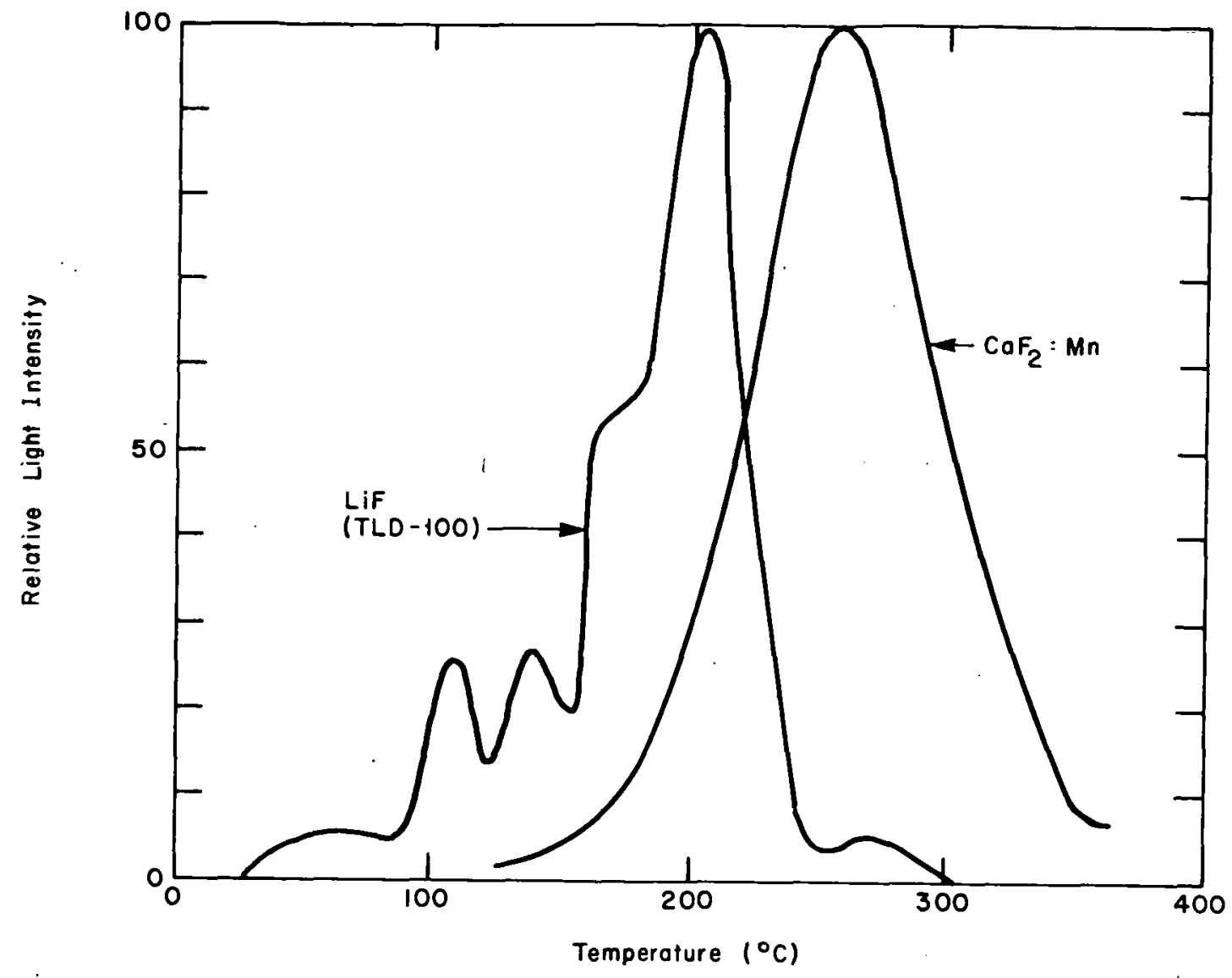

Fig. 2 Relative light intensity of the thermoluminescent phosphors $\mathrm{CaF}_{2}: \mathrm{Mn}$ and $\mathrm{LiF}$ (TLD-100) as a function of phosphor temperature.

When the radiation spectrum is narrow, a photomultiplier tube can be used which has nearly the same sensitivity for all the wavelengths in the spectrum. However, when the radiation spectrum is broad, the response of the photomultiplier tube to all the wavelengths in the band would not be the same. Thus, a correction would generally have to be made, accounting for these sensitivity differences.

Generally, an optical filter is used between the photomultiplier tube and the TL phosphor which is being heated in the thermoluminescent reader on a planchet. This filter blocks out any infrared radiation emitted by the heated planchet. Thus, if the TL phosphor emits radiation in the infrared region, the infrared will be filtered out and this portion of the signal from the TL phosphor will be lost. This would affect the correspondence of the integrated reading from the TL phosphor to the absorbed dose.

\subsection{Retention of Stored Signal}

The retention of the stored signal by the phosphor is another important characteristic. LiF is reported to lose only $5 \%$ of its stored signal in a year ${ }^{[2]}$. However, $\mathrm{CaF}_{2}: \mathrm{Mn}$ loses about 20 to $30 \%$ of its signal in the first 24 hours, and an additional 5 to $10 \%$ in the next seven days. The signal losses from both types of phosphors in a given period of time are believed to vary with each batch of phosphor produced by a manufacturer and also from manufacturer to manufacturer. The large fading rate of the $\mathrm{CaF}_{2}: \mathrm{Mn}$ stored signal introduces a problem in correlating the stored signal in the phosphor to the absorbed dose. Generally, a number of $\mathrm{CaF}_{2}: \mathrm{Mn}$ dosimeters are calibrated against a standard gamma source and are read out approximately 48 to 72 hours after irradiation (period of time after irradiation in which the rate of fading is small). This establishes a relationship between the readout and the known absorbed dose for this time after irradiation. After irradiation in an unknown gamma source, the $\mathrm{CaF}_{2}:$ Mn dosimeters are read out for 


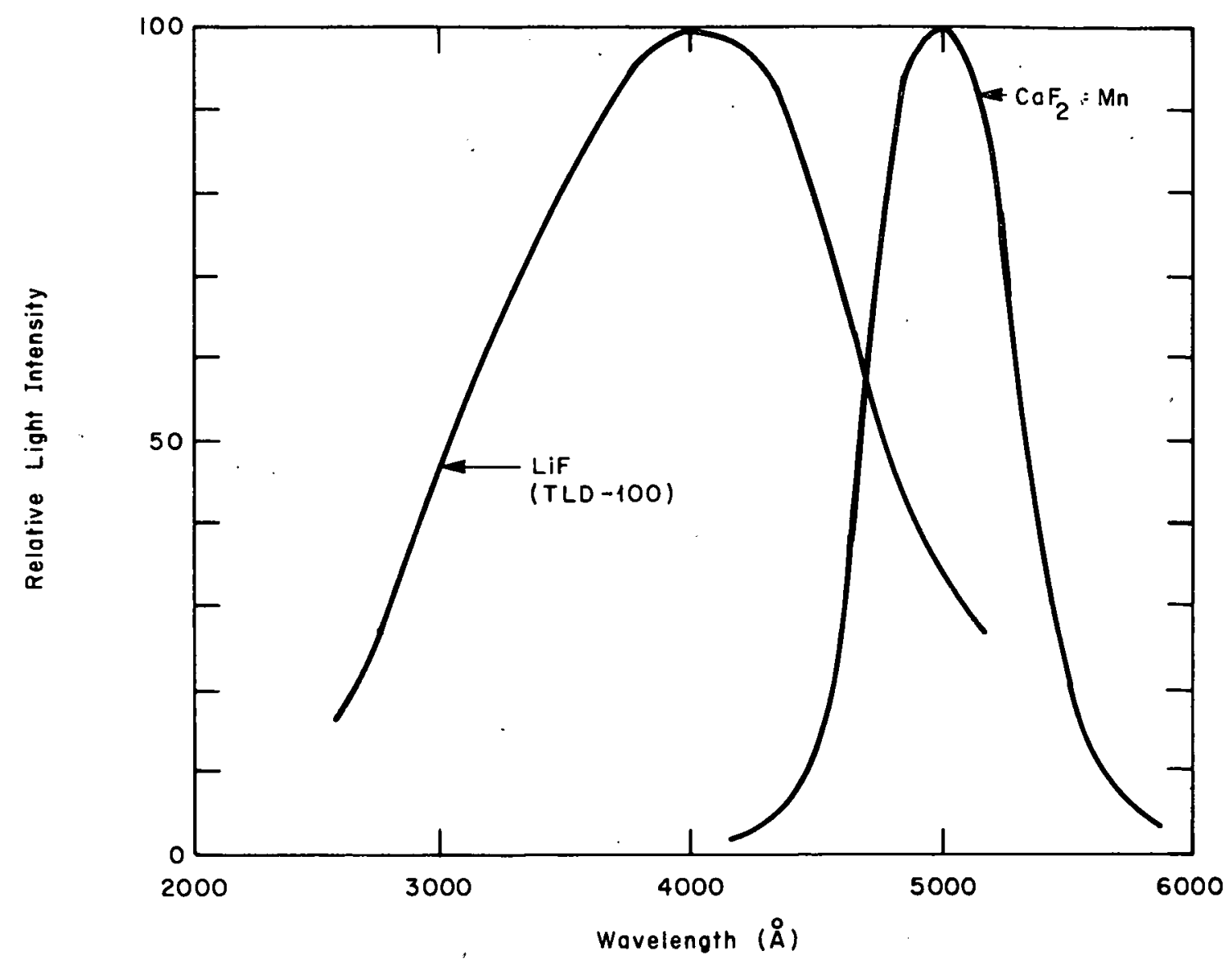

Fig. 3 Relative light intensity of the thermoluminescent phosphors $\mathrm{CaF}_{2}: \mathrm{Mn}$ and LiF (TLD-100) as a function of emitted radiation wavelength.

the same time after irradiation as the $\mathrm{CaF}_{2}: \mathrm{Mn}$ dosimeters calibrated against the standard source. This procedure automatically corrects for the large fading rate of the stored signal for the $\mathrm{CaF}_{2}: \mathrm{Mn}$; thus an accurate determination of the absorbed dose by the dosimeters in an unknown radiation field can be made.

\subsection{Response as a Function of Incident Gamma Energy}

The response of TL phosphors to gamma radiation as a function of gamma energy is dependent on the mass energy absorption coefficient of the phosphor. In turn, the mass energy absorption coefficient is dependent on the cross section of the material for the photoelectric, Compton, and pair production effects. Since the cross section for each of these effects is dependent on the atomic number, the energy response of the different phosphors is a function of their effective atomic numbers. Figure 4 shows the response per roentgen of $\mathrm{CaF}_{2}: \mathrm{Mn}$ and $\mathrm{LiF}$ normalized to unity for the effective gamma energy of ${ }^{60} \mathrm{Co}(1.25 \mathrm{MeV})^{[2]}$. The much greater response of $\mathrm{CaF}_{2}: \mathrm{Mn}$ for gamma energies. less than $0.1 \mathrm{MeV}$ compared to that for $\mathrm{LiF}$ is due to:

(1) The larger effective atomic number of $\mathrm{CaF}_{2}: \mathrm{Mn}$

(2) The predominance of the photoelectric effect in this energy region

(3) The cross section per atom for the photoelectric effect which varies as $Z^{4}$ (where $\mathrm{Z}$ is the atomic number). 


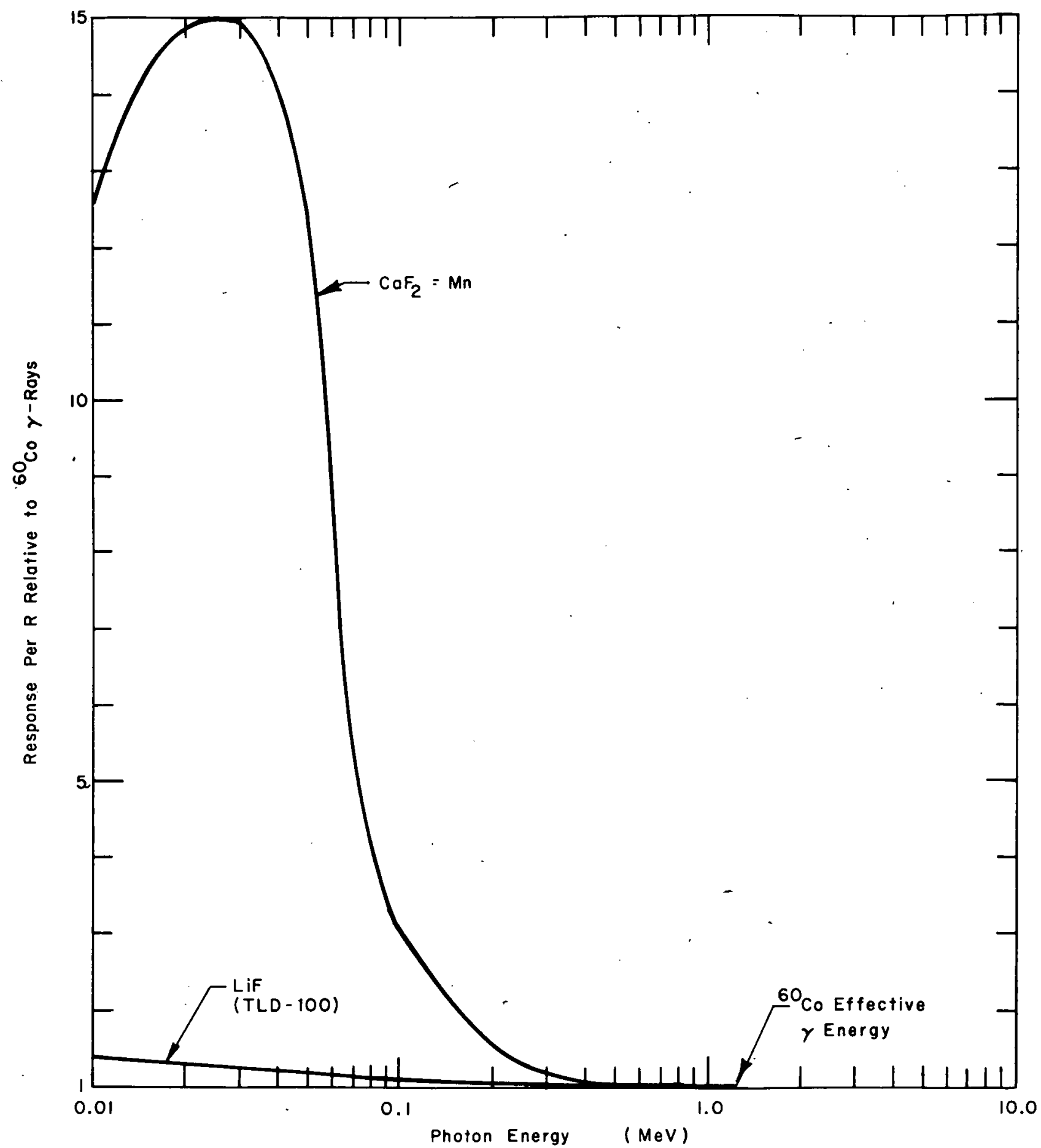

Fig. 4 Relative response per roentgen of the thermoluminescent phosphors $\mathrm{CaF}_{2}: \mathrm{Mn}$ and LiF (TLD-100) as a function of gamma energy. Both curves are normalized to 1 at the effective gamma energy of $1.25 \mathrm{MeV}$ for ${ }^{60} \mathrm{Co}$. 


\subsection{Response to Thermal Neutrons}

Thermoluminescent phosphors are used to determine the intensity of a gamma field. However, often the gamma radiation is accompanied by other radiations, in particular, neutrons. Thus the response of different TL phosphors to neutrons is one response characteristic that is commonly investigated. Since LiF (TLD-100) contains a small percentage of ${ }^{6} \mathrm{Li}$ which has a large thermal neutron absorption cross section, some response is expected from thermal neutrons. It has been reported that for a thermal neutron fluence $\left(\mathrm{n} / \mathrm{cm}^{2}\right)$ of $10^{11}$ the response was measured to be approximately equivalent to $5 \times 10^{3}$ rads of $60 \mathrm{Co}$ gamma radiation [3]. The response of $\mathrm{CaF}_{2}: \mathrm{Mn}$ to thermal neutrons in the same measurement could not be accurately determined but was estimated to be equivalent to 10 to $20^{60} \mathrm{Co}$ rads for a fluence of $1.5 \times 10^{12}$ $\mathrm{n} / \mathrm{cm}^{2}$.

Thus when desiring to measure only the dose of the gamma field in a thermal neutron and gamma mixed radiation field, the contribution to the stored signal of the TL phosphor from the thermal neutrons will have to be made. When the intensity of the neutron and gamma fields are about the same, the correction for thermal neutrons to the stored signal for $\mathrm{CaF}_{2}: \mathrm{Mn}$ is small, but quite significant for $\mathrm{LiF}$ (TLD-100).

\subsection{Response of Phosphor as a Function of Exposure}

The response of TL phosphors as a function of gamma dose is another important characteristic. Figure 5 shows the response of $\mathrm{CaF}_{2}: \mathrm{Mn}$ and $\mathrm{LiF}$ (TLD-100) as a function of exposure ${ }^{[2]}$. These curves show that $\mathrm{CaF}_{2}: \mathrm{Mn}$ has a linear response as a function of exposure. The upper part of the curve where the curve bends over reflects the exposure limit of the phosphor. The curve for LiF is linear over the lower exposure range and then changes slope at approximately $10^{3}$ roentgen. The curve is then linear again until it reaches its dose limit. This knee in the LiF exposure curves introduces an inconvenience in that more calibration data are needed with $\mathrm{LiF}$ than for $\mathrm{CaF}_{2}: \mathrm{Mn}$.

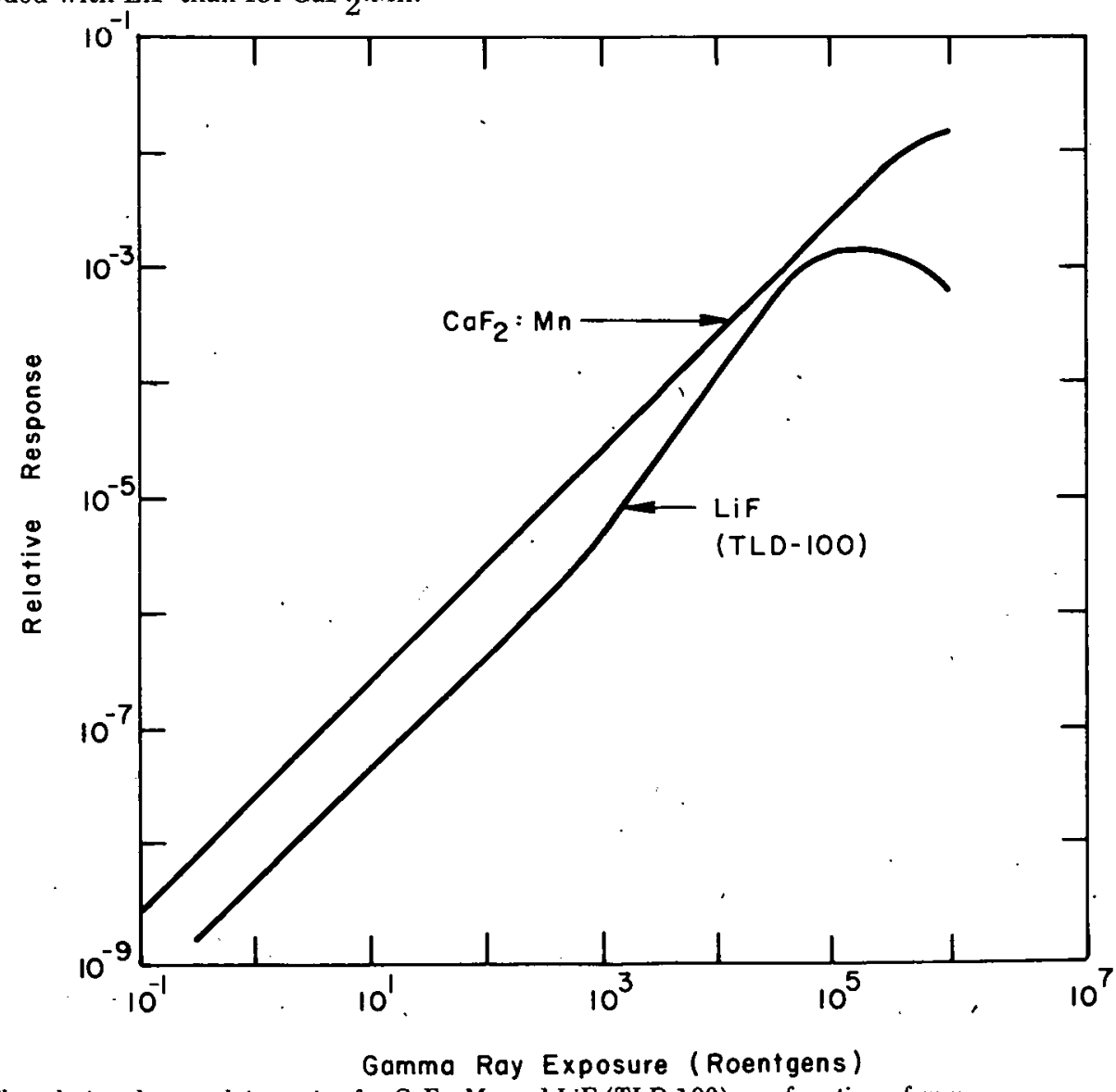

Fig. 5 The relative glow peak intensity for $\mathrm{CaF}_{2}: \mathrm{Mn}$ and LiF (TLD-100) as a function of gamma-ray exposure. 


\section{THERMOLUMINESCENCE MEASURING APPARATUS}

A reader for thermoluminescent phosphors generally consists of (a) a heating device to heat the phosphor, (b) a photomultiplier tube to measure the luminescence given off by the phosphor, and (c) the electronics necessary to display the PM tube output in the desired form. Figure 6 shows these components of a typical TL reader $[2]$.

The first step in reading a TL phosphor is placing the phosphor (which is either pressed into a geometric form or exists as a loose powder) onto a heating tray (planchet). A current is then passed through the planchet which heats the planchet and in turn heats the phosphor. Generally, the atmosphere around the phosphor is nitrogen to eliminate any spurious luminescence resulting primarily from the presence of oxygen. As the phosphor heats up it luminesces. This luminescence is detected by the PM tube. The output from the PM tube is amplified and displayed on a recorder. In addition, the temperature as a function of time of the planchet is generally displayed on the recorder. Often a reader will include the electronics necessary to integrate the output from the PM tube and display this quantity on a digital display. This display or the integration of the area under the glow curve on the recorder represents the number of electrons that were trapped in the phosphor during irradiation. This is also a measure of the absorbed dose during irradiation.

Many other innovations often included in a commercial reader which were not discussed above provide a more accurate reading from the TL phosphor which then more accurately represents the absorbed dose of the phosphor from the irradiation.

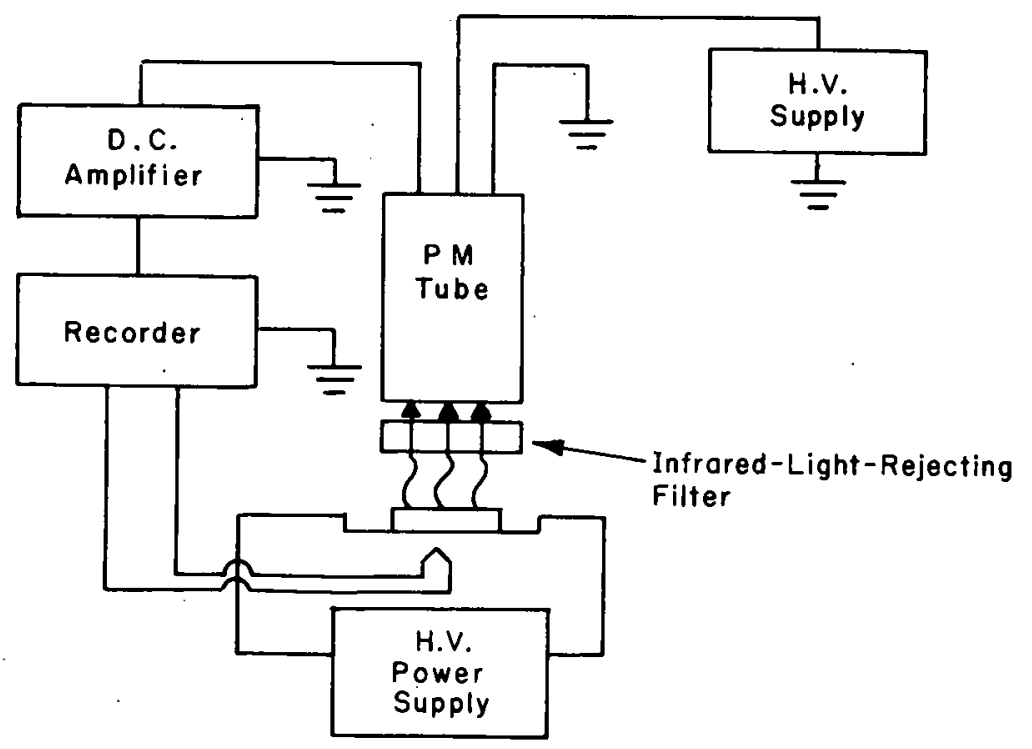

Fig. 6 Schematic diagram of the principal components of a thermoluminescent reader. 


\section{APPLICATION OF THERMOLUMINESCENT DOSIMETRY TO MEASURING GAMMA HEAT}

Before the principles of thermoluminescent dosimetry can be applied to making gamma heat determinations in a critical facility, it is first necessary to define gamma heat and then to determine qualitatively if thermoluminescent dosimetry can be used to make an accurate and useful gamma heat determination. Set forth below are: (a) a definition of gamma heat, (b) a qualitative discussion of charged particle equilibrium, (c) the calibration of the readout of a TL dosimeter to gamma heat, and (d) the interpretation of the readouts of TL dosimeters (after irradiation in a critical facility) that are not shrouded with charged particle equilibrium material.

\section{QUANTITATIVE DESCRIPTION OF GAMMA RAY ENERGY DEPOSITION}

The actual dissipation of gamma energy in matter takes place in two steps. In the first step, gamma rays interact with atomic electrons of matter through the photoelectric and Compton effects or create electrons as a result of the pair production effect. In these interactions the gamma rays impart some or all of their energy to the electrons. This energy transfer can be determined quantitatively by using the following expression:

$$
\mathrm{K}=\int_{\mathrm{E}_{1}}^{\mathrm{E}_{2}} \varphi(\mathrm{E}) \frac{\mu_{\mathrm{en}}}{\rho}(\mathrm{E}) \mathrm{dE}
$$

where

$\varphi(\mathrm{E})$ is the gamma ray flux as a function of the gamma ray energy $\frac{\mu_{\mathrm{en}}}{\rho}(\mathrm{E})$ is the mass energy absorption coefficient as a function of gamma ray energy for
the material of interest.

This expression is referred to as the kerma and has units of ergs/gram.

The next step is the tranfer of energy from the secondary electrons to matter through coulomb-force interactions with other electrons. This interaction is described quantitatively by:

$$
\dot{\mathrm{D}}=\frac{\Delta \mathrm{E}_{\mathrm{D}}}{\Delta \mathrm{m}}
$$

where

$\Delta \mathrm{E}_{\mathrm{D}}$ is the energy imparted to matter in the small mass, $\Delta \mathrm{m}$.

The quantity $D$ is the absorbed dose and has units of ergs/g or rads (where $1 \mathrm{rad}=100 \mathrm{ergs} / \mathrm{g}$ ).

Thus, the actual deposition of the gamma-ray energy in matter occurs in the second step and the quantity of interest in gamma heat measurements is the absorbed dose. In calculating gamma heat at a point of interest in a piece of material, the kerma is the expression that is used since it is more readily determined. Since there is rarelyia one-to-one correspondence between the kerma and the absorbed dose at any point in a medium, there is obviously an error in determining gamma heat from the kerma. However, in a measuring device (such as a calorimeter or Bragg-Gray chamber) the energy deposition of the secondary electrons (ie, absorbed dose) is measured and thus the gamma heating is determined directly. 


\section{CHARGED PARTICLE EQUILIBRIUM}

There is one condition in a medium in which the kerma is nearly the same as the absorbed dose. This condition is described as charged particle equilibrium (ie, CPE). As stated by Attix [4] "... this is usually described as the balance of incoming and outgoing electrons at the boundary of a specifed volume. An alternative, and more useful, way of thinking of it is as the equality of the absorbed dose and the kerma at a point of interest." Thus when a CPE condition exists the value of the kerma is nearly the same as the absorbed dose.

When irradiating a small solid state dosimeter in air with a gamma source, it is easily seen that some of the electrons created in the dosimeter will leak out and be lost in the air. If the incoming electrons (that are created in the medium and stopped in the dosimeter) do not compensate for the energy losses from the electrons leaking out of the dosimeter, the absorbed dose in the dosimeter will be different than the kerma. It is possible to correct for this effect by shrouding the dosimeter with a material that has nearly the same average stopping power for the secondary electrons and mass absorption coefficient as the dosimeter material. The thickness of this material should be sufficient to stop the most energetic secondary electron created in the surrounding medium. This buffer material will now provide an incoming electron energy flux which is nearly equivalent to that leaving the dosimeter, thus setting up a balance of incoming and outgoing electrons. If this is the case, the computed kerma for the dosimeter will be nearly the same as the dose absorbed by the dosimeter. (The kerma is never actually the same as the absorbed dose because actual deposition of energy from the electrons created in a small volume of material takes place outside the volume -- for all practical purposes this difference is negligible.)

\section{CALIBRATION OF DOSIMETER RESPONSE TO GAMMA HEAT}

A standard gamma source is used for calibrating a dosimeter to gamma heat. Generally, one of two commonly known sources, ${ }^{137} \mathrm{Cs}$ or ${ }^{60} \mathrm{Co}$, is used. The calibration dosimeters shrouded with CPE material are then exposed to this source for a given exposure $[\mathrm{a}]$. Knowing the gamma spectrum of the standard source and the exposure given the dosimeters, the kerma of the dosimeters can be determined using Equation (1). Since either one of the above gamma sources can be considered essentially a source of monoenergetic gamma rays the kerma equation can be simplified to read:

$$
\mathrm{K}_{\text {mat }}=\varphi(\mathrm{E}) \frac{\mu_{\text {en }}}{\rho_{\text {mat }}}(\mathrm{E}) \mathrm{E}
$$

Since CPE material was used around the dosimeter, the kerma is nearly equivalent to the absorbed dose and, thus, Equation (2) becomes

$$
\mathrm{D}_{\text {mat }}=\varphi(\mathrm{E}) \frac{\mu_{\mathrm{en}}}{\rho_{\mathrm{mat}}}(\mathrm{E}) \mathrm{E}
$$

where $D_{\text {mat }}$ is the absorbed dose in ergs/g. The definition of roentgen yields an important equivalence. The absorbed dose in air per roentgen of exposure is $86.9 \mathrm{ergs} / \mathrm{gram}$, independent of the gamma energy. Thus the absorbed dose in air for the calibration is

Solving for $\varphi(\mathrm{E}) \mathrm{E}$ gives

$$
\left.\mathrm{D}_{\text {air }}(\text { ergs } / \text { gram })=\varphi(\mathrm{E}) \frac{\mu_{\text {en }}}{\rho_{\text {air }}}(\mathrm{E}) \mathrm{E}=\mathrm{X} \text { (roentgens }\right) \cdot 86.9
$$

$$
\varphi(\mathrm{E}) \mathrm{E}=\frac{\mathrm{X} \text { (roentgens) } \cdot 86.9}{\frac{\mu_{\mathrm{en}}}{\rho_{\mathrm{air}}}(\mathrm{E})} .
$$

[a] Exposure is given in units of roentgens only. 
Substituting this expression for $\varphi(\mathrm{E}) \mathrm{E}$ in Equation (3) gives

$$
\mathrm{D}_{\text {mat }}\left(\text { ergs/gram) }=X(\text { roentgens }) \cdot 86.9 \cdot \frac{\frac{\mu_{\text {en }}}{\rho_{\text {mat }}(E)}}{\frac{\mu_{\text {en }}}{\rho_{\text {air }}}(\mathrm{E})}\right.
$$

Multiplying Equation (6) by $10^{-7}$ converts the units to $\frac{\text { watt-sec }}{\text { gram }}$. Thus the expression for the number of watt-sec absorbed per gram of material is

$$
\mathrm{D}_{\text {mat }}\left(\frac{\text { watt-sec }}{. \text { gram }}\right)=X(\text { roentgens }) \cdot 86.9 \cdot \frac{\frac{\mu_{\text {en }}}{\rho_{\text {mat }}}(E)}{\frac{\mu_{\text {en }}}{\rho_{\text {air }}(E)}} \cdot 10^{-7} .
$$

Thus, knowing (a) the exposure given the dosimeters, (b) the effective entergy of the gamma source, and (c) the mass energy absorption coefficients for air and the dosimeter material for that gamma energy, the absorbed dose received by the dosimeter can be computed.

By reading the dosimeter, the readout can be correlated with the computed absorbed dose for the dosimeter from Equation (7) to give the calibration factor. By multiplying the readout of dosimeters exposed in unknown gamma fields by this factor, the absorbed dose from the gamma field in the dosimeter material is determined. Knowing the time of exposure and dividing this time into the absorbed dose obtained from Equation (7) gives the average gamma heat rate of the unknown field in the dosimeter material.

\section{INTERPRETATION OF DOSIMETER READOUTS AFTER IRRADIATION IN A CRITICAL FACILITY}

Generally, in-core gamma heat measurements are required in positions where space for a dosimeter is very limited. Consequently, in order to preserve the environment around the point of measurement (ie, by not removing any reactor hardware in order to accommodate a dosimeter), the size of the dosimeters is kept at a minimum. And because of lack of space, there is not enough room for CPE material to be placed around the dosimeter. Thus the dosimeters are placed in the reactor bare.

The mediums surrounding the bare dosimeter in the reactor will generally not provide charged particle equilibrium for the dosimeter. However, since it is assumed that the readout of the dosimeter is proportional to the dose absorbed by the dosimeter, the existence of CPE is unimportant in this case. Thus the dosimeter should indicate the absorbed dose in the material and for the thickness of the dosimeter. In terms of gamma heat the dosimeter would indicate an average surface gamma heating rate for the time of exposure and for the material of the dosimeter at the point of measurement.

If the surrounding mediums did provide charged particle equilibrium and the surrounding medium had nearly the same mass absorption coefficient and average stopping power for the secondary electrons as the dosimeter material, then the gamma heat measured by the dosimeter would be that of the surrounding medium at the location of the dosimeter. If the medium and the dosimeter material were dissimilar under CPE conditions, the dosimeter readout could be used to determine gamma heat for two different mediums one of which would be that of the dosimeter material. In this case the readout of the dosimeter would be interpretated in the same manner as the readout from the dosimeter where CPE did not exist. It would also be possible to determine the gamma heat in terms of the surrounding medium at the location of the 
dosimeter. Knowing the gamma heat of the dosimeter material (determined in the manner described above) and solving an expression which is a modified form of the Bragg-Gray relationship, the gamma heat in terms of the surrounding material can also be determined ${ }^{[5]}$. This expression requires knowledge of the secondary electron and gamma-ray fields in the surrounding medium. The modified Bragg-Gray relationship is used because generally the dosimeter is too large to permit the use of the unmodified Bragg-Gray relationship.

Since solid state dosimeters are responsive to beta radiation and beta radiation is present in a reactor radiation field, part of the dosimeter readout will be due to this beta radiation. The intensity of the beta radiation field is generally not accurately known throughout the reactor. Thus, a correction for the contribution to the dosimeter readout from this radiation is not readily made. Therefore, the readout of the dosimeter in this case indicates both gamma and beta heating. 


\section{TECHNIQUE FOR MEASURING GAMMA HEAT IN A CRITICAL FACILITY}

Much effort was expended in arriving at a suitable thermoluminescent dosimeter material to measure gamma heat in a critical facility. The thermoluminescent phosphor $\mathrm{CaF}_{2}: \mathrm{Mn}$ was chosen based on (a) a comparison between gamma heat measurements in ATR and thermoluminescent phosphor gamma heat measurements in the ATR critical facility, and (b) the negligible response of the phosphor to thermal neutrons.

A number of gamma heat measurements have been made in the critical facilities and on at least one occasion have been compared to thermocouple gamma heat measurements in the test reactor. This comparison showed the two gamma heat results to be in excellent agreement.

Some of the problems associated with this technique are (a) the fading of the $\mathrm{CaF}_{2}: \mathrm{Mn}$ stored signal, (b) extrapolation of the gamma heat results from the critical facility to its respective test reactor, and (c) reduction of the relative error for the $\mathrm{CaF}_{2}: \mathrm{Mn}$ dosimeters.

This technique for measuring gamma heat in a critical facility using $\mathrm{CaF}_{2}: \mathrm{Mn}$ dosimeters is discussed in the following section of this report.

\section{1. $\mathrm{CaF}_{2}: \mathrm{Mn}$ THERMOLUMINESCENT PHOSPHOR CHARACTERISTICS}

The thermoluminescent phosphor chosen for use in the critical facilities to measure gamma heat was $\mathrm{CaF}_{2}: \mathrm{Mn}$. The form of the phosphor is a square bar of solid $\mathrm{CaF}_{2}: \mathrm{Mn}$ measuring $1 \times 1 \times 6 \mathrm{~mm}$. The high sensitivity for gamma rays, the low neutron sensitivity, the independence of the response as a function of dose rate, and the broad dose range of this phosphor make it very useful for in-core absorbed dose determinations. Some of the representative characteristics for this phosphor are shown in Table I. These characteristics will vary slightly from manufacturer to manufacturer and with different batches of the phosphor from the same manufacturer.

TABLE I

\section{NOMINAL CHARACTERISTICS OF SOLID $\mathrm{CaF}_{2}$ :Mn DOSIMETERS}

Dimension:

Dose Range:

Dose Rate Dependence:

Energy Dependence

Temperature:

Fast Neutron Response:

Thermal Neutron Response:

Linearity:
$1 \times 1 \times 6 \mathrm{~mm}$

$\sim 10 \mathrm{mR}-10^{6} \mathrm{R}$

Less than $10 \%$ to $10^{11} \mathrm{R} / \mathrm{sec}$

See Figure 4

Negligible Dependence at Ambient Temperature

$\sim 1 \times 10^{-9} \mathrm{R} / \mathrm{n} / \mathrm{cm}^{2}$

$\sim 1 \times 10^{-10} \mathrm{R} / \mathrm{n} / \mathrm{cm}^{2}$

Within $3 \%$ over total dose range 


\section{IRRADLATION TECHNIQUE}

The technique for irradiating the $\mathrm{CaF}_{2}: \mathrm{Mn}$ dosimeters in a critical facility is to first locate the dosimeter in the reactor where gamma heat is to be measured. If the reactor power distribution and power level are needed for normalizing the gamma heat results on the basis of power, fission rate monitors should also be loaded. The reactor is then loaded for the conditions under which the gamma heat measurements are to be made (ie, simulated xenon equilibrium poison, experiment loadings, etc). The reactor is then raised to a power level of approximately 200 to 300 watts in a predetermined power division. The duration of the irradiation is 20 minutes. The timing of the irradiation begins when the reactor power level reaches $1 / \mathrm{e}$ of the maximum power level for the irradiation. The timing of the irradiation is shown in Figure 7.

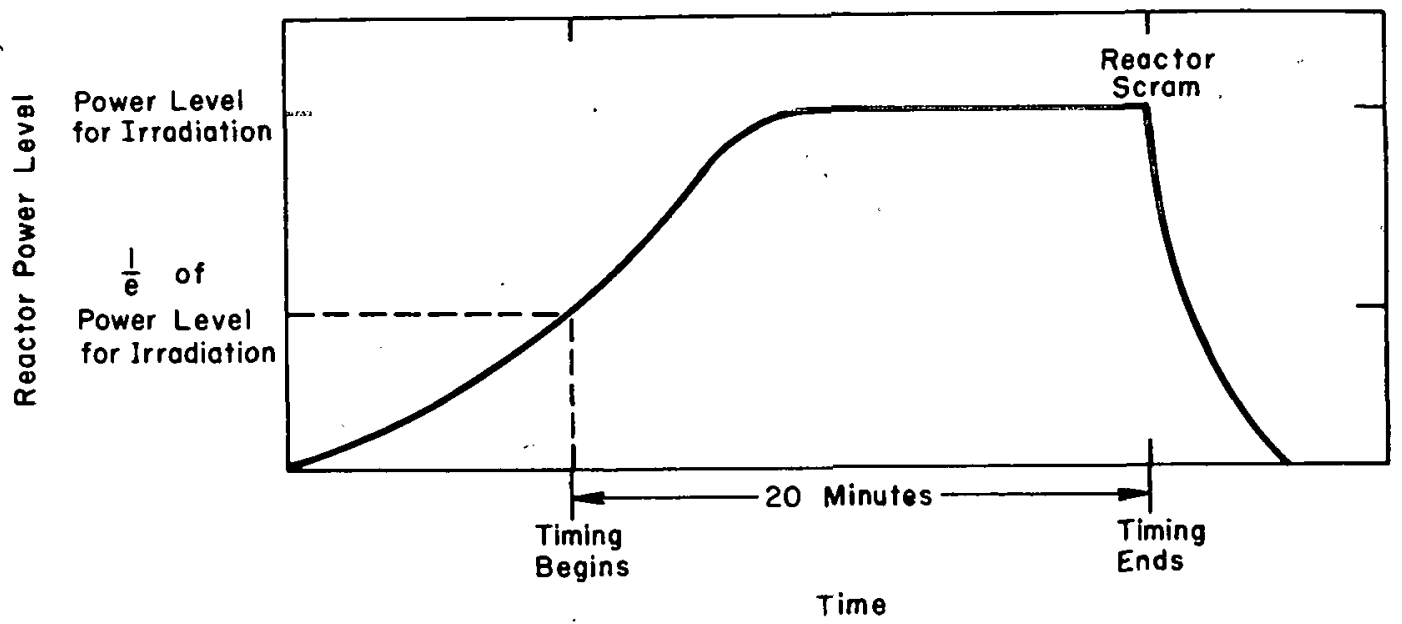

Fig. 7 Illustration of the timing of a flux run to assure that the exposure to the dosimeters in the reactor is equivalent to the exposure if the flux run were made at the maximum power level for the time of the irradiation.

This timing will assure that the gamma exposure from the irradiation is equivalent to a 20 -minute gamma exposure at the maximum power level for the irradiation. As soon after the scram as possible, the $\mathrm{CaF}_{2}: \mathrm{Mn}$ dosimeters are removed from the shutdown gamma field existing in the reactor.

\section{CORRECTIONS TO $\mathrm{CaF}_{2}: \mathrm{Mn}$ DOSIMETER READOUTS}

After the dosimeters have been read out and before the readouts of the $\mathrm{CaF}_{2}: \mathrm{Mn}$ dosimeters can be used to represent an equivalent test reactor measured gamma heat, several corrections have to be made. These corrections are those due to (a) the fading of signal of the phosphor (if not already made), (b) the delayed gammas, not reaching equilibrium during irradiation, and (c) the exposure due to the shutdown gamma field. These corrections are discussed below.

\subsection{Fading of Stored Signal}

Presently, the calibration dosimeters are read out for the same wait time after exposure as the dosimeters that were irradiated in the critical facility. This procedure asssures that both types of dosimeters have the same fade and no corrections are necessary for the loss of signal. Both the irradiated and the calibrated dosimeters should be from the same batch and the same manufacturer, and both should have the same radiation history to ensure that the dosimeters have the same fade characteristics. 


\subsection{Correction for Time-Varying Fission Product Source Strength}

The gamma core source is divided into two groups, the prompt-fission gamma and the fission product gamma sources. During the 20 -minute irradiation in the critical facility, the prompt-fission gamma source has the same source strength as would be found in the test reactor for the same power level. However, the fission product gamma source does not reach equilibrium in the 20-minute irradiation in the critical facility. Thus the exposure to the thermoluminescent dosimeters from the fission product gammas is less than that found in the test reactor which has been operating for a long period of time for the same power level. Since the maximum heating effect from the reactor gamma sources is to be measured, a correction has to be made for the fission product gamma source not at equilibrium.

The prompt-gamma energy release per fission is $7.9 \mathrm{MeV} /$ fission. The fission product gamma energy release per fission after a long period of operation is $7.2 \mathrm{McV} /$ fission. The fission product cncrgy rclcase for a 20 -minute irradiation has been calculated to be $4.48 \mathrm{MeV} /$ fission [6]. Thus the core source from the critical facility irradiation is $12.38 \mathrm{MeV} /$ fission as compared to $15.1 \mathrm{MeV} /$ fission if the fission product gamma source were at equilibrium. This difference in core source is alleviated when the dosimeters are left in the critical facility shutdown gamma field after scram.

Since the time the dosimeters are left in the critical facility after scram varies, the correction for this exposure will vary. A particular example for the exposure due to the shutdown gamma field is for when the dosimeters were left in the critical facility 12 minutes after scram and then removed. The average energy per fission due to the shutdown gamma field is $0.68 \mathrm{MeV} /$ fission. Thus the total average energy release for the irradiations and exposure to the shutdown gamma field is $13.06 \mathrm{MeV} /$ fission and the correction factor to be applied to the gamma heat data obtained from thermoluminescent dosimeters is 1.16 (ie, 15.1/13.06). It is worthy to note that this correction is dependent on the irradiation time: The above correction was for a 20-minute irradiation.

Corrections for a range of irradiation and shutdown exposure times are not presently available. Thus the irradiation must be for 20 minutes and the dosimeters removed from the reactor approximately 12 minutes after scram in order to use the correction factor of 1.16 .

\subsection{Correction for Distance from Core Source}

Since the thermoluminescent dosimeters are generally measuring gamma dose rates in a region of the reactor not directly adjacent to the fuel, the previously quoted correction for the core varying gamma source strength is not entirely applicable to the dosimeters. This is due to the fact that away from the fuel the gamma source is partly from the fuel and partly from the capture gammas ( $\eta, \gamma$ reactions) in materials around the dosimeter as a result of inelastic neutron scattering and neutron capture in these materials. Since the percentage contribution from the fuel source varies with distance from the core and the type of adjacent materials, a percentage contribution from the fuel would have to be determined for each dosimctcr. However, assuming only one percentage contribution for all dosimeters located within an experiment loop is expected to have a negligible effect on the accuracy of the measurement. It will be assumed that $66 \%$ of the gamma source for the dosimeters located within an experiment loop is from the fuel. Thus the correction factor for the fission product gammas not having reached equilibrium during the 20 -minute irradiation and compensated for the exposure to the shutdown gamma field is 1.10 [ie, $1+$ $(0.16)(0.66)=1.10]$.

Corrections for dosimeters located away from the fuel and not in an experiment loop have not been evaluated. Thus corrections for these dosimeters will have to be evaluated on an individual basis. 


\section{DISCUSSION OF ERRORS}

In an attempt to determine the precision and accuracy of using $\mathrm{CaF}_{2}: \mathrm{Mn}$ dosimeters in determining gamma heat in the test reactor from an irradiation in the respective critical facility, some of the errors and their magnitudes are discussed in the following sections. In addition an estimate of the relative and absolute errors of this technique for measuring gamma heat is derived.

\section{SOURCE OF ERRORS}

In order for this dosimeter material to be useful as a gamma heat detector in a mixed radiation field, it is desirable for the dosimeter material to have negligible response to the radiations other than gamma. Two other radiations which are of significant intensity in a reactor radiation field are neutrons and betas. Since beta radiation should cause the same response in the dosimeter material as secondary electrons, the stored signal of the dosimeter from the beta radiation can be quite significant when the beta field is significant. Corrections for the contribution of the betas to the stored gamma signal of the $\mathrm{CaF}_{2}: \mathrm{Mn}$ dosimeter are not readily made because the intensity of the beta field throughout the reactor is not known. Therefore, when the beta field around the dosimeter is significant, the readout of the dosimeter should be interpreted to represent heating effects due to both gammas and betas.

The possible effects to the stored signal of $\mathrm{CaF}_{2}: \mathrm{Mn}$ from neutrons are more indirect because it is not an ionizing radiation. The different effects neutrons might have on $\mathrm{CaF}_{2}: \mathrm{Mn}$ and the significance of these effects are discussed below. In addition, the possible errors introduced during the calibration of the $\mathrm{CaF}_{2}: \mathrm{Mn}$ dosimeters to gamma heat, and during the readout process, are also discussed.

\subsection{Neutron Effects ${ }^{[a]}$}

The exposure of $\mathrm{CaF}_{2}: \mathrm{Mn}$ to neutrons in a mixed radiation field can lead to many undesirable effects when desiring to measure only the gamma dose of this field. These effects will be divided into two groups, direct and indirect. The direct effect is defined as the neutron activation of the different constituents of $\mathrm{CaF}_{2}: \mathrm{Mn}$ which lead to the emission of heavy ionizing particles. These emitted particles will then interact with the phosphor and create trapped electrons. Thus the neutrons interacting with the phosphor will add to the gamma signal and give an indication of a higher gamma heating rate when the dosimeter is read. The indirect effect is defined as the neutron interactions with the phosphor other than activation which affect the signal normally stored by the phosphor from the gammas.

The direct effects on $\mathrm{CaF}_{2}: \mathrm{Mn}$ from exposure to neutrons are through the capture of neutrons by the constituents of the phosphor. The capture of thermal neutrons by the doping agent, manganese, is the only thermal neutron capture event by the phosphor which leads to emission of a heavy ionizing particle. The emitted particle in this case is a $2.9-\mathrm{MeV}$ beta. An experiment was conducted to determine the sensitivity. of the phosphor to this event. The conclusion was that the contribution to the gamma signal stored by the phosphor in a mixed radiation field (typical of light water reactors) is considered to be insignificant as compared to the precision of the measurement. The cross section for the ${ }^{19} \mathrm{~F}(\eta, a){ }^{16} \mathrm{~N}$ reaction is only significant for neutron energies greater than $3 \mathrm{MeV}$. The ${ }^{16} \mathrm{~N}$ created by this event decays with a 7.3-second half-life. The decay involves the release of $10.4 \mathrm{MeV}$ of energy through the release of betas, gammas, and neutrinos. The contribution of signal to the gamma signal in a light water reactor radiation field from this event is also considered to be insignificant.

[a] This is a summary of a more detailed discussion which can be found in Reference 7. 
The indirect effects on $\mathrm{CaF}_{2}: \mathrm{Mn}$ from neutron exposure are electron trap emptying and creation and the energy deposition of the recoil nuclei from neutron scattering events.

Crystal lattice dislocations in $\mathrm{CaF}_{2}: \mathrm{Mn}$ can act as electron trapping centers. These dislocations can be created by "the recoil of a nucleus upon emission of a gamma ray following thermal neutron capture, or upon an elastic interaction with an energetic neutron." An experiment conducted to determine the magnitude of this effect showed that the effect was not distinguishable within the precision of the measurement.

Fast neutrons have the capacity of dumping trapped electrons. Thus a portion of the stored signal from gammas would be destroyed by this effect. An experiment investigating this trap dumping effect showed it to be indistinguishable within the uncertainty of the medsurement.

\subsection{Calibration Errors}

The arrangement of the apparatus to establish a relationship hetween thermoluminescence of the phosphor and gamma heating is illustrated in Figure 8.

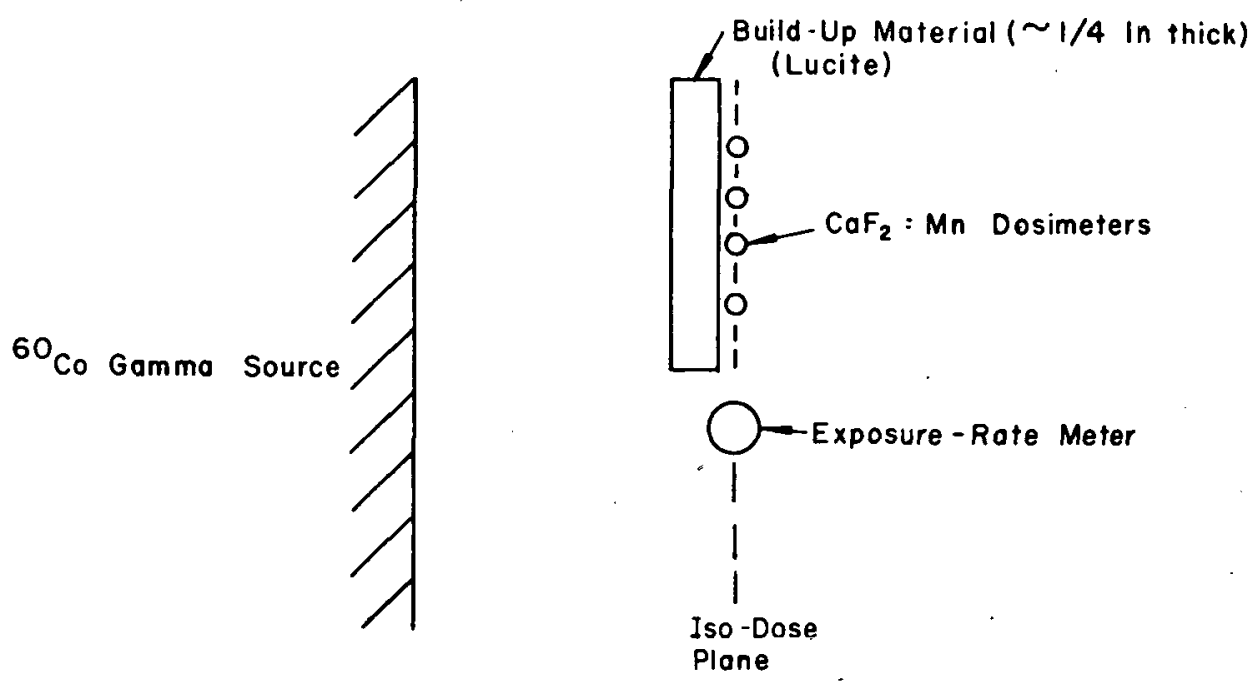

Fig. 8 Illustration of physical setup for calibrating the thermoluminescence of $\mathrm{CaF}_{2}: \mathrm{Mn}$ to absorbed dose.

Some of the errors that may be introduced during the calibration are:

(1) An inequality in the exposure of the dosimcters and that indicated by the exposure-rate meter

(2) Exposure differences between dosimeters

(3) Incorrect build-up geometry and material.

The dosimeters to be calibrated are placed in the same iso-dose plane as the center of the exposure-rate meter. The iso-dose planes were determined in the absence of the lucite build-up material. Thus it is assumed that the same attenuation of the gamma source exists over the build-up material for the dosimeters as for the build-up material around the chamber.

Exposure differences between each of the calibration dosimeters would also introduce an error into the calibration. However, since all the dosimeters are placed in the same iso-dose plane, this error is expected to be negligible. 
Since the dosimeters during the calibration have only a build-up material between the source and the dosimeters, it is uncertain as to whether this is the correct geometry for the build-up material. In order to determine the effect of different build-up geometries and the difference between using lucite and aluminum as a build-up medium, a number of $\mathrm{CaF}_{2}: \mathrm{Mn}$ dosimeters were exposed to a ${ }^{60} \mathrm{Co}$ source with five different build-up configuration. In two configurations, the dosimeters were sandwiched between two pieces of lucite and aluminum. These configurations mocked up the situation where each individual dosimeter is shrouded by either aluminum or lucite in order to achieve charged particle equilibrium at all surfaces of the dosimeter. Two other configurations had buildup material only on the side of the dosimeter facing the source. The fifth configuration had no buildup material between the source and the dosimeters.

The average readouts of each group of dosimeters in the first four configurations, which were all exposed to the same source for the same period of time, compared within the precision of the measurement. This comparison indicates that charged particle equilibrium material need only be placed against the dosimeters on the side facing the collimated source and it is not necessary to place CPE material on both sides of each dosimeter for this particular calibration setup.

The fifth configuration was an attempt to determine the difference in readouts of dosimeters with CPE material placed between the dosimeters and source with no build-up medium other than air. However, the results showed that the photoelectrons emanating from the lead collimator (normally absorbed by the lucite or aluminum build-up material in the case of the other four configurations) caused the readouts of these dosimeters to be double that for the dosimeters exposed in the four other configurations. Thus, a conclusion as to the difference in absorbed dose for the dosimeters for having air or lucite as a buildup medium was not possible.

\subsection{Reading Errors}

Errors that may be introduced during the reading of the $\mathrm{CaF}_{2}: \mathrm{Mn}$ dosimeters from the calibration and irradiation in the critical facility are:

(1) The dosimeter surface may be contaminated with contaminants which may luminesce during the reading

(2) Planchet luminescent during the reading

(3) Incorrect thermoluminescent analyzer setup

(4) Poor heat transfer from the planchet to the dosimeter.

Dosimeters which are suspected of being contaminated must be cleaned in an acetone solution or in an ultrasonic cleaner. Commonly, dosimeters are attached on reactor hardware by adhesive tape. After the tape is removed from the dosimeter after irradiation, there is often a residue from the tape adhesive left on the dosimeter. The adhesive will luminesce during the readings and this luminescence will be added to that normally given off by the phosphor. By either cleaning the dosimeters or sealing the dosimeter in polyethylene bags during irradiation, this and other contamination problems are eliminated.

Since the planchet is heated to temperatures around $400^{\circ} \mathrm{C}$, the planchet gives off infrared radiation and would contribute to the luminescence given off by the phosphor. Most of this luminescence is filtered out by the infrared filter. However, since there is a small amount of luminescence passed by the filter, the planchet is checked periodically for its luminescence. The planchet luminescence is checked by putting the planchet through a heating cycle without a dosimeter. In this way, a glow curve is generated for the planchet. This glow curve can then be compared to that typically produced by the phosphor. If the area of the planchet glow curve is negligible as compared to that of the phosphor, the planchet luminescence can be neglected. If not, a constant correction factor will have to be applied to each phosphor glow curve (since 
the luminescence of the planchet should be constant for each heating-cycle). Typically the planchet luminescence is negligible when reading dosimeters with 1000 roentgens of exposure or more.

The settings of the thermoluminescent analyzer are recorded for each group of dosimeters read. These settings may vary with each type of phosphor but are the same whenever the same types of phosphor are read. This practice of recording settings of the TL analyzer minimizes errors introduced by incorrect settings. Table A-I of Appendix A gives the typical settings for a TL analyzer for reading solid $\mathrm{CaF}_{2}: \mathrm{Mn}$ dosimeters $(1 \times 1 \times 6 \mathrm{~mm})$. Additionally, the absence of the nitrogen purge during the readout would result in spurious luminescence. This effect can be detected from the glow curve. Thus a check of the glow curve after each readout would result in the detection of this abnormality and could also indicate any other abnormality that might affect the dosimeter readout.

Varying heat transfer characteristics between dosimeter and planchet affects the dumping of the trapped electrons. In order to get a good correlation between the absorbed dose of the dosimeter and the thermoluminescent output from the dosimeter it is necessary to dump nearly all the trapped electrons and have the heat transfer characteristics between the dosimeter and planchet constant for each dosimeter being read. Heat transfer changes are generally a result of dimensional variances in the dosimeters. Since the same planchet is used to retain the dosimeters during the heating process, dimensional variances in the dosimeters cause surface contact changes with the planchet. This causes variations in the amount of heat transferred to the dosimeter. This in turn affects the amount of signal dumped from the dosimeter; thus the indication of the dose received by the dosimeter during the irradiation will be in error. Experience has shown that when the heat transfer between the dosimeter and planchet is inadequate, the dose indicated by the dosimeter is lower than the true value. This discrepancy can be as large as 30 or $40 \%$.

In order to lessen the effect of this error on the results of a gamma heat measurement, the practice has been to locate two or three dosimeters at each measurement point. Then if this heat transfer problem affects some of the dosimeter readouts, this will be apparent in the discrepancy between the values of the dosimeters at each measurement point. The incorrect data point has always proven to be the smallest value of the values of the dosimeters at the suspected data point. (The time of heating and readout has been. subsequently lengthened in order to reduce this source of error.)

\section{DETERMINATION OF RELATIVE AND ABSOLUTE ERRORS}

A determination of the absolute and relative errors in the measurement is necessary in order to determine the value of the gamma heat values obtained from this gamma heat measuring technique. The magnitude of the errors previously discussed will be estimated, if not previously measured, and then combined to make a determination of the relative and absolute errors for this measuring technique.

\subsection{Relative Error}

The magnitude of the relative error is important in determining the uncertainty in the relative distribution of gamma heat values obtained from this gamma heat measuring technique. This is of particular interest in determining thermal bowing on a reactor component which results from gamma heat gradients across the reactor piece.

The errors which affect the relative error of the measurement are mainly due to errors occurring in the reading process. These would include the random errors such as (a) fluctuating conditions in the reader, (b) varying heat transfer properties between the dosimeters and the planchet, and (c) variations in phosphor or activator content in the dosimeters. 
An estimate of the magnitude of the relative error was made by irradiating a large number of dosimeters to the same exposure and then determining the distribution of the readouts of these dosimeters. It was determined that the error at the $95 \%$ confidence level was $\pm 20 \%$. To reduce the relative error of the measurement, dosimeters to be used in the critical facilities are preselected from a batch of dosimeters that were exposed to a source, and readout, and a standard deviation of the distribution of the readouts calculated along with the mean readout value for the distribution. The dosimeters that are used are those that are within $\pm 5 \%$ of the mean value of the distribution. This preselection process eliminates dosimeters which are irregular in weight or other physical properties which would affect their response to gammas relative to the dosimeters which have nearly identical physical properties. Thus, a reliable estimate of the relative error as a result of this preselection process is $\pm 5 \%$ at a confidence level of $95 \%$. Errors affecting the relative error of the measurement which would not be included in the preselection process are considered to be negligible.

Since these dosimeters are re-usable, the dosimeters are also grouped as to the similarity of their radiation history. Thus, dosimeters to be used for a particular gamma heat run should have nearly the same radiation history. This minimizes any errors that may be introduced because of sensitivity changes due to differing radiation exposures, neutron fluences, and number of times annealed.

\subsection{Absolute Error}

The absolute error of this technique for measuring gamma heat is important in determining the reliability of the absolute gamma heat values. This is important in determining cooling requirements for different reactor components in the test reactors based on the gamma heat values determined from the irradiation of the $\mathrm{CaF}_{2}: \mathrm{Mn}$ dosimeters in the critical facilities.

Contributing errors which are considered to affect the absolute error of this measurement are:

(1) the relative error

(2) neutron effects

(3) inaccuracy of calibration of dosimeter readout to gamma heat in aluminum

(4) inequality in energy response of aluminum and $\mathrm{CaF}_{2}: \mathrm{Mn}$ to gammas

(5) inaccuracy in technique for corrections due to exposure to shutdown gamma field

(6) inaccuracy in technique for corrections due to time-varying gamma sources during the irradiation.

In an effort to arrive at an absolute error for this gamma heat measuring technique the errors associated with each of the perturbations given above are estimated below:

\section{Perturbation}

Relative error

Neutron effects

During calibration
Estimated Error For 95\% Confidence Level

$\pm 5 \%$

$\pm .5 \%$

$\pm 2 \%$ 
Inaccuracy of calibration

Inequality of energy response

Shutdown corrections

Delay gamma buildup corrections

Combined Error for 95\% Confidence Level $\pm 10 \%$

$\pm 15 \%$

$\pm 5 \%$

$\pm 5 \%$

$\pm 21 \%$

Thus the estimated absolute error for this measurement is $\pm 21 \%$ at a confidence level of $95 \%$. With the errors considered, the absolute error represents the uncertainty in the gamma heat values for the test reactor obtained from a measurement in its respective critical facility assuming no differences in the two reactors. It is worthwhilc to note that the above error does not include any errors occurring as a result of normalizing the gamma heat data based on reactor power. Additionally, the absolute error does not include any effect associated with the response of $\mathrm{CaF}_{2}: \mathrm{Mn}$ to beta since the gamma heat values oblained lrom the dosimeters are meant to represent heating effects from both gamma and beta. There is also an uncertainty associated with the readout of the dosimeter correctly indicating the absorbed dose from the betas because the calibration does not establish the absolute beta sensitivity. This uncertainty is also not included in the absolute error. 


\section{CONCLUSIONS}

Determining gamma heating in a test reactor from the irradiation of $\mathrm{CaF}_{2}: \mathrm{Mn}$ dosimeters in the test reactor critical facility has presently fulfilled the requestor's needs for gamma heat information. However, in the future more demands will probably be made of this technique for gamma heat information because of its ability to obtain spatially detailed gamma heat distributions.

Before more demands for gamma heat information can be expected, confidence in this technique has to be established. This can be achieved by intercomparison of data obtained from this technique with that measured in the test reactor or calculated from gamma heat codes. Some difficulty arises in this latter comparison. When using a gamma heat code, the gamma heat values are determined for charged particle equilibrium conditions, whereas the dosimeters in the critical facilities are irradiated bare, and in most cases they have not satisfied charged particle equilibrium requirements. Thus there is always this discrepancy in the two values. An experiment could be devised such that charged particle equilibrium conditions exist for the dosimeter and thus a more direct comparison between the two methods could be made.

If the magnitude of the contributing errors to the absolute uncertainty for this gamma heat technique could be better defined, the confidence in this technique would be further established.

The ability of this technique to measure gamma heat in other reactor materials (such as stainless steel) is needed. Presently, the requestor converts the measured gamma heating in aluminum to another material based on the ratio of the atomic numbers of the two materials (providing the atomic number of the other material is close to that for aluminum). This assumes that the gamma energy spectrum at the point of measurement in the reactor is confined to the energy band where the Compton effect is dominant.

The application of the Bragg-Gray theory to in-core gamma heat measurements would provide gamma heat information for other materials. This would require the shrouding of a small piece of dosimeter material with the material in which gamma heating is to be measured. The shroud must be thick enough to provide charged particle equilibrium and the thickness of the dosimeter material has to be small as compared to the range of the secondary electrons created in the shroud. It is worthwhile to mention that these gamma heat detectors (shroud and dosimeter) would be restricted as to their use in the reactor because of their size.

The ability to determine the gamma heat gradient across the wall of a reactor in-core piece would be a further refinement in this measuring technique. This would allow for an accurate determination of the cooling requirements for this in-core piece. 


\section{REFERENCES}

1. J. H. Schulman, "Survey of Luminescence Dosimetry", in U.S. Atomic Energy Commission, Techuical Information Division, Luminescence Dosimetry, AEC Symposium Series-8 (April 1967) pp 11-12.

2. F. H. Attix and W. C. Roesch, Radiation Dosimetry, Volume II, New York and London: Academic Press, 1966, pp 269-283.

3. A. R. Reddy et al, "Thermoluminescence Response of LiF to Reactor Neutrons", Radiation Research, 40 (1969) pp 552-562.

4. F. H. Attix, "Basic X-ray Dosimetry", Health Physics 15 (1968) pp 49-56.

5. F. H. Attix and W. C. Roesch, Radiation Dosimetry, Volume I, New York and London: Academic Press, 1966, pp 363-368.

6. B. H. Alsop, Bettis Atomic Power Laboratory, private communication (May 1971).

7. G. S. Sanford et al, Determination of Gamma-Ray Heating in a Critical Facility By Thermoluminescent Dosimetry, ANL-7373 (February 1968). 


\section{APPENDIX A \\ PROCEDURE FOR USING CaF ${ }_{2}$ :Mn DOSIMETERS TO MEASURE \\ GAMMA HEAT IN A CRITICAL FACILITY}

Given below is a typical procedure for determining gamma heat for a test reactor from irradiating $\mathrm{CaF}_{2}: \mathrm{Mn}$ dosimeters in its respective critical facility.

(1) Determine the number of solid $\mathrm{CaF}_{2}: \mathrm{Mn}$ dosimeters $(1 \times 1 \times 6 \mathrm{~mm})$ to be used for the gamma heat measurement. It is suggested that three dosimeters be located at each data point to assure the integrity of the measured gamma heat value.

(2) Obtain the number of $\mathrm{CaF}_{2}: \mathrm{Mn}$ dosimeters needed for the measurement. Make sure that these dosimeters all have nearly the same radiation history and have been recently annealed.

(3) Arrange for the calibration dosimeters to be exposed to a standard ${ }^{60} \mathrm{Co}$ source at the same time the dosimeters in the reactor are exposed. The exposures should cover the range of the exposures expected from the dosimeters exposed in the critical facility. A suitable buildup material should be present between the source and calibration dosimeters such that (a) the most energetic electron created in the medium between the source and the build-up material is stopped in the build-up and (b) the electron energy flux in the build-up material is nearly the same as that if the build-up material was the same as the dosimeter material. Typically an irradiation in a critical facility in an experiment position at 300 watts for 20 minutes will give an exposure of 2000 roentgens.

(4) Load dosimeters into the reactor making sure the exposure to the shutdown gamma field is negligible compared to the exposure during the irradiation. If not negligible then the shutdown field exposure should be determined so that a correction to the readout of the dosimeters can be made.

(5) Irradiate dosimeters for 20 minutes at a reactor power. level of approximately 300 watts. Corrections for delay gamma buildup for irradiations other than 20 minutes have not presently been determined.

(6) Remove dosimeters from the reactor approximately 700 seconds after the scram terminating the 20 minute flux run.

(7) Record the time after scram each dosimeter is removed from the reactor in order to correct the dosimeter readout for the shutdown gamma field exposure after scram if different than $\mathbf{7 0 0}$ scconds.

(8) Read the critical facility and calibration dosimeters for a wait time of at least 48 hours after exposure. Both the critical facility and calibration dosimeters should be read for the same wait time after exposure. Table A-I shows the settings of a typical TL analyzer for the optimum readout of the solid $\mathrm{CaF}_{2}: \mathrm{Mn}(1 \times .1 \times 6 \mathrm{~mm})$ dosimeters.

(9) Convert the dosimeter readings from the irradiation in the critical facility to equivalent roentgens based on the calibration curve of readout versus roentgen derived from the calibration dosimeters. 
(10) Correct the roentgen equivalent value for each dosimeter for exposure to the shutdown gamma field before (if significant) and after the irradiation.

(11) Correst the roentgen equivalent value for each dosimeter for the delay gamma source not at equilibrium during the irradiation. This correction should take into account the distance of the dosimeter from the core source.

(12) Convert the equivalent roentgen for each dosimeter to watts/gram of aluminum using the conversion factor $6.985 \times 10^{-9} \mathrm{w} / \mathrm{g}$. This factor is for a 20 minute irradiation and for the use of a ${ }^{60} \mathrm{Co}$ gamma source during the calibration. Each dosimeter now represents gamma heat in aluminum at the point of measurement with the delay gamma source at equilibrium.

TABLE A-I

TYPICAL TL ANALYZER CONDITIONS FOR READING OF SOLID

$$
\mathrm{CaF}_{2}: \mathrm{Mn}(1 \times 1 \times 6 \mathrm{~mm}) \text { DOSIMETERS }
$$

$\begin{array}{ll}\text { Nitrogen Planchet Cool } & - \text { Yes } \\ \text { Nitrogen Purge } & - \text { Yes } \\ \text { Planchet Type } & - \text { Silver } 1 / 4 \text { in. Depression } \\ \text { High Voltagc (V) } & -750 \\ \text { Reading Cycle (sec) } & -60 \\ \text { Temperature Rate } & -\sim 8^{\circ} \mathrm{C} / \sec \text { (for } \sim 38 \text { seconds) } \\ \text { Preheat Planchet } & -100^{\circ} \mathrm{C} \text { (in } 4 \text { seconds) } \\ \text { Maximum Temperature } & -400^{\circ} \mathrm{C} \text { (for } \sim 18 \text { seconds) }\end{array}$


